\title{
Proceeding
}

Supplementary Issue: Winter Conferences of Sports Science. Costa Blanca Sports Science Events, 22-23 March 2021. Alicante, Spain.

\section{Artificial musculature: An overview of the different exoskeleton models and their applications}

EMANUELA ISABELLA FERRARO , FRANCESCA OROFINO, MARIA CHIARA PARISI, MARIA BELLOMO, VINCENZO CRISTIAN FRANCAVILLA, CATERINA CRESCIMANNO

Faculty of Human and Social Sciences, University of Enna "Kore", Italy

\begin{abstract}
Recovery of upper and lower limbs function is essential to reach independence in patients with neurodegenerative diseases. The exoskeleton is an external wearable structure that provide to increase the physical capabilities with important applications in medical and rehabilitative therapy. Robotic exoskeletons for upper and lower limbs, based on the principle of motor learning, have been introduced in neurorehabilitation. The treatment must be individualized according to the characteristics of the patient, to provide him all the help necessary for the improvement of his quality of life. Wearable exoskeletons have potential for a number of applications including early rehabilitation, promoting physical exercise, and may improve mobility and independence, moreover, may reduce secondary health conditions related to sedentariness, with all the advantages that this entails. This work focuses on the importance of using the exoskeleton for the rehabilitation of people with neurodegenerative diseases and problems related to loss of motor skills, highlighting the benefits of this type of rehabilitation compared to traditional physical therapy. In conclusion, these devices represent an important turning point in the lives of many disabled people for their independence but also for their assistance. The hope for the future is to be able to permanently replace the wheelchair.
\end{abstract}

Keywords: Exoskeleton; Rehabilitation; Neurodegenerative diseases.

\section{Cite this article as:}

Ferraro, E.I., Orofino, F., Parisi, M.C., Bellomo, M., Francavilla, V.C., \& Crescimanno, C. (2021). Artificial musculature: An overview of the different exoskeleton models and their applications. Journal of Human Sport and Exercise, 16(3proc), S892-S904. https://doi.org/10.14198/ihse.2021.16.Proc3.06

Corresponding author. Faculty of Human and Social Sciences, University of Enna "Kore", Italy.

E-mail: emanuelaisabella.ferraro@unikorestudent.it

Abstract submitted to: Winter Conferences of Sports Science. Costa Blanca Sports Science Events, 22-23 March 2021. Alicante, Spain.

JOURNAL OF HUMAN SPORT \& EXERCISE ISSN 1988-5202.

(c) Faculty of Education. University of Alicante.

doi:10.14198/jhse.2021.16.Proc3.06 


\section{INTRODUCTION}

The exoskeleton is an external cybernetic device able to enhance the physical abilities of the subject that is covered, constituting a sort of "artificial musculature".

These are designed to assist people with locomotion problems, to increase the physical abilities of those who for work reasons are forced to transport or lift heavy loads, to rehabilitate patients after debilitating surgery, but especially in patients with severe neurological disorders.

To date, most research has focused on the development of equipment exoskeletons for the rehabilitation of survivors of strokes, to individuals with traumatic injuries spinal cord (SCl), and, to military personnel to increase their combat capabilities and improve their mobility.

The use of the exoskeleton is increasingly widespread and several studies have focused on the also focus on the psychological impact that the use of the exoskeleton may have on patients with motor problems.

It is already known, how much the motivation and in general the psychological state of the subject influence on the success of rehabilitation. With the use of the exoskeleton the subject implements and controls the movement of a specific joint in a unit of time.

According to the principles of neuroplasticity and motor learning, robotic devices were introduced into rehabilitation so that they can interpret the afferent input from the joints and provide specific stimulation to the central nervous system to promote recovery and carry out daily activities independently.

When a robotic movement is done, in addition to the typical motor areas, brain areas such as the insula, amygdala and other neural circuits connected to the centres are activated deeper.

These centres are important for the memory of the movement and the motivation of the gesture. So, the use of robotic instruments can induce a series of sensory processes that through the stimulation can be stored in the central nervous system (Molteni et al., 2018).

In this short review the contribution of the exoskeleton in the field of pathologies will be examined neurological, highlighting the benefits of this type of rehabilitation compared to therapy traditional physics.

The use of the exoskeleton allows an intensive training, for this reason, is the most convenient type of exercise to assess the level of recovery of strength and the exact execution of movement patterns.

\section{METHOD}

To examine and compare the different types of exoskeletons, a literature search was carried out in order to reconstruct history and development.

The first robotic devices made their debut in the 70s, when Miomir Vukobratovic from the Mihailo Pupin Institute in Belgrade produced an active exoskeleton for the lower limbs.

This exoskeleton was hydraulic and allowed the flexion and extension of the hip, the knee and ankle, as well as the abduction and adduction of the hip. 
At the same time, Seireg and Grundman at the University of Wisconsin planned an exoskeleton to facilitate walking, to sit, to get up, to climb and downstairs. Also, this exoskeleton was hydraulic.

The desired movement was chosen by the patient using switches, while the trajectory final movement was pre-programmed in the device, mimicking that of a subject normal (Simeonov \& Veneva, 2019).

The models recently made include detectors of electrical impulses that record the neural or neuromuscular impulses of the operator and which transmit them to automatic devices in order to move the mechanical part of the exoskeleton.

Modern exoskeletons are equipped with different sensors such as accelerometers, measuring units inertial (IMU), EMG, EEG, pressure sensors, which allow us to measure various physical characteristics such as angles of junction, position, ground contact, pressure, force exercised.

These sensors are essential for this device to best assist the subjects who wear it.

As already mentioned, exoskeletons can be used for different purposes: they can increase the performance of a healthy subject, or they can be assistance devices for patients with motor diseases.

There are 3 types of systems that activate this type of devices: electric motor, hydraulic actuators and pneumatic.

Exoskeletons can also be distinguished into:

- Active: they provide active assistance to the movement of the patient;

- Passive: provide non-motorised support to patients;

- Active-assisted: they complete the movement after the user has started it;

- Resistive: they offer opposition to the execution of the subject's movement;

- Interactive: allow correction of movements (Simeonov \& Veneva, 2007).

Depending on the part of the body they support, exoskeletons can be classified as: Upper limb, lower limb (LEE) exoskeletons, whole body exoskeletons and specific joint support.

Studies carried out by several universities, as well as research institutes and industrial companies have carried out constant research in this field and in recent years have been devised different types of exoskeletons (Simeonov \& Veneva, 2019; Bing Chen et al., 2015).

Ortesi Lokomat was developed in 2001 by the company Hocoma (Zurich, Switzerland), for the rehabilitation of the path; provides support to patients with motor limb dysfunction lower. The Lokomat system (Figure 1a) consists of a robotic orthosis for locomotion, a body weight support system and a treadmill. The patient exercises in an environment virtual reality with constant audio and visual feedback.

S. K. Banala et al. 2013, of the University of Delaware (Newark, DE, USA), have developed the Active Leg Exoskeleton (ALEX) for the rehabilitation of gait patients with motor disabilities.

The treatment can be adapted to the needs of patients with selective movement control at the level of the joints. It is easy to wear and does not need any calibration complex, in fact, thanks to its sensors, ALEX is ready for use from the ignition. 
ALEX offers different degrees of freedom, allowing support and movement of the joints: coxo-femoral, knee and ankle.

The Ekso exoskeleton (Figure 1b) developed by Ekso Bionics (Richmond, CA, USA) in 2005 is designed for the care and rehabilitation of patients with varying levels of limb weakness lower. Has a variable assistance, according to the needs of the subject. Ekso is a device battery powered that is worn over clothing and shoes and is maneuverer with the hand movements.

It has four electric motors, a steel and carbon structure with 15 computerized motion sensors, thanks to which it recognizes the intentions of the user in real time and calculates and performs the corresponding movements. Batteries have a lifetime of four hours and the exoskeleton can be used in two modes: the first is the one with the aid of a remote control and with the help of a physiotherapist to coordinate movements, usually in a first phase of use must be assisted, then the patient can walk alone, helped by two smart crutches.

Rewalk exoskeleton, developed by Rewalk Robotics (Marlborough, MA, USA) in 2008 provides an enhanced hip and knee movement to enable people with spinal cord injury of standing and also walking. This exoskeleton is controlled by an on-board computer and by motion sensors allows you to walk exploiting the inclination of the upper part of the body. It has motors positioned on the hips and on the knees. The engines are driven by a software that is located in a computer that you put on shoulders like a backpack. The communicator clock serves to activate the exoskeleton. The person who Wear Rewalk by moving the centre of gravity of the body back and forth orders the computer to move the legs and generate a sequence of steps. This exoskeleton is used with crutches.

The Vanderbilt exoskeleton developed by M. Goldfarb et al., in 2012 is a LEE that allows paralyzed patients to perform basic movements. Its total weight is $12 \mathrm{~kg}$. The diet is supplied by sophisticated motors in the knee and hip joints and, in combination with sensors advanced and control strategies, the device allows people with walking problems to get up and walk again, ensuring them a new level of independence at home and in the community. It can also be used in domestic and community environments.

About two decades ago, a research group from the University of California Berkeley (Berkeley, CA, USA) began working in the field of exoskeletons. The Berkeley Lower Extremity Exoskeleton (BLEEX) (Figure 1c) was developed to help soldiers carry loads heavy; allows to carry with little effort loads of about $35 \mathrm{~kg}$ with only $4 \mathrm{I}$ of diesel for the power supply of the hydraulic system, over a duration of about 3 hours of autonomy. Highlights of this system are the 15 degrees of freedom (Simeonov \& Veneva, 2019), that is the number of different movements possible from the exoskeletal suit. Degrees of freedom are an essential parameter in that ensure manoeuvrability and portability to the model.

BLEEX wearers can walk at an average speed of $1.3 \mathrm{~m} / \mathrm{s}$, from this group other exoskeletons have also been developed, such as: ExoHiker, ExoClimber and Human Universal Load Carrier.

Hybrid Assistive Limb (HAL) (Figure 1d) was developed by the University of Tsukuba a Tsukuba, Japan, for different applications such as: helping healthy people to improve their strength and help people with mobility disorders to perform essential movements of the everyday life. HAL 3 and HAL 5 were developed in 2012.

HAL- 5 represents the fifth version of HAL, weighs $23 \mathrm{~kg}$ and is powered by electric motors. HAL- 5 gives the possibility to the subjects who wear it to lift heavy objects up to $70 \mathrm{~kg}$. 
The HAL-3 variant was created to assist older people in locomotion, this device is regularly used in various Japanese hospitals. It can work with 3 different modes. The most innovative of them detects and amplifies the signals that the patient tries to send to specific muscles and assists them in movement.

Hal is able to pick up the wearer's nerve signals through a series of electrodes applied to the lower limbs.

Unlike other "traditional" exoskeletons, which only passively produce the step in one direction, at a predefined pace in a completely automatic way, Hal through electrodes positioned on the patient's skin at the lower level, it captures and analyses the nerve signals that the brain receives from the muscles and responds by providing adequate assistance e thus allowing fluid and natural movements.

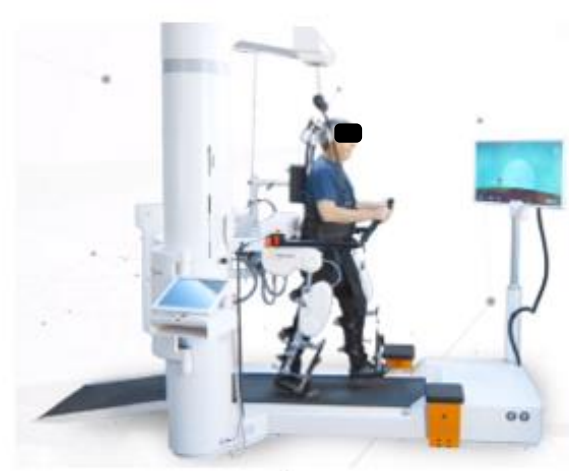

a.

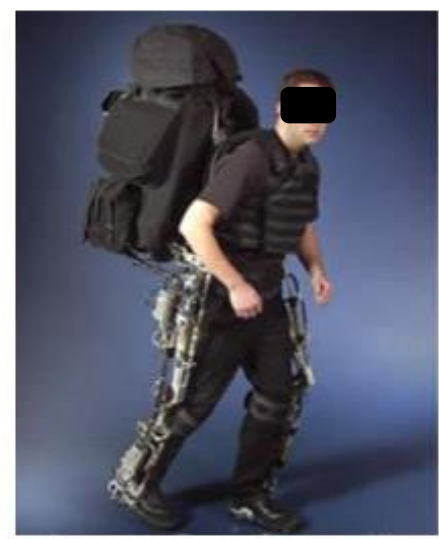

c.

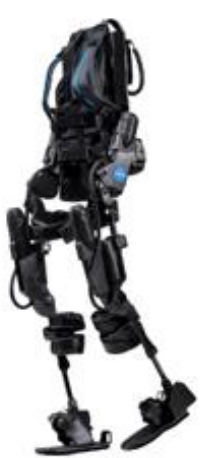

b.

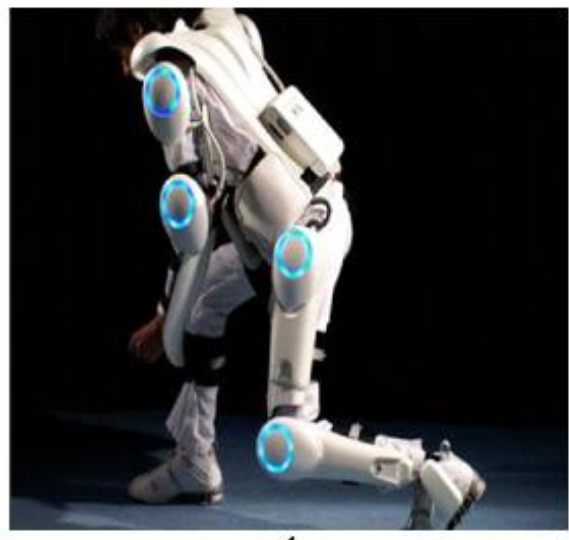

d.

1a: Lokomat, Hocoma; 1b: Ekso, Ekso Bionics; 1c: BLEEX, University of California Berkeley; 1d: HAL, Tsukuba University.

Figure 1. Different types of exoskeletons developed and manufactured by private companies and universities.

The LOPES exoskeleton was developed by the University of Twente in 2012 (Enschede, Countries Bassi) as a locomotion rehabilitation device. Its peculiarity, compared to simple traditional braces, is that it allows you to set eight different degrees of freedom for the joints, customizing the therapy for each patient. It allows patients to spend more time practicing regaining their mobility, and at the same time yes reduces the need for health personnel to assist in the rehabilitation process.

ANdROS exoskeleton was developed in 2011 by Harvard Medical School (Cambridge, MA, USA) and Spaulding Rehabilitation Hospital (Charlestown, MA, USA) as a tool wearable for gait rehabilitation. The 
frame is made of aluminium along with the joints to make them light and powerful. It is equipped with handles to support the torso.

There are also robotic devices designed to assist operators, in fact, it was developed a device by the Kanagawa Institute of Technology in Atsugi, Japan, for help nurses to transfer patients, so as to avoid operators making efforts without strain the muscular, neurological and bone systems too much. The exoskeleton can be configured for provide varying degrees of care, in order to help the patient according to his or her needs for therapy fixed. The subject can also set the support for each leg differently, adapting it depending on the case and thus allowing the recovery of different types of patients. This technology it can also be used by nurses, physiotherapists. One of the examples of assisted exoskeleton is "AUTONOMYO". This device is designed for the elderly and those suffering from diseases neuromuscular. Autonomyo is a multi-function exoskeleton. To allow total freedom of movement, has 6 motorized axes at the hips and knees and six passive axes at the height of the ankles. The exoskeleton allows you to perform complex movements very similar to a natural walk.

The Autonomyo team is also working on intention and augmentation detection balance that would allow a symbiosis of movement assisted by the device, as well as a higher security level.

Multiple sensors for movement, force and inertia are processed approximately 500 times for second with advanced algorithms to overcome these challenges. The exoskeleton currently weighs $25 \mathrm{~kg}$ and yes stands up on its own, which means that the user does not perceive absolutely the weight. It can also be adjusted for heights from $160 \mathrm{~cm}$ up to $200 \mathrm{~cm}$. for the assistance and transport of patients.

Another robotic aid called APO (Active Pelvic Orthosis), in 2017 was designed for help people in locomotion. The device weighs approximately $5 \mathrm{~kg}$. The sensors installed on it they detect inclinations and are activated automatically. APO is able to understand when a-senior is about to fall to the ground and reacts accordingly. Based on the weight of the wearer and the movement of the limbs, the exoskeleton is able to act in just 350 milliseconds and create a force contrary which brings the patient back to his feet in a position from which it is difficult for him to fall again. The machine can be calibrated in just three minutes and does it all by itself. The wearer must not undergo any type of training.

EXPOS is another of the devices developed in 2015 by Sogang University for the aid of movement of older people, has a total weight of $3 \mathrm{~kg}$ and heavier parts such as the motor, battery and controller are positioned on a rotating swivel; it's an exoskeleton motorized with a carbon and aluminium frame. This exoskeleton allows the use of the movement and provides greater safety and autonomy to users.

Among the aged care exoskeletons, we still find several devices such as ad example the WWH (Wearable Walking Helper), the WPAL (Walking Power Assist Leg) and ReStore also used by patients who are in the post-stroke phase (Kapsalyamov \& Jamwal, 2019).

\section{RESULTS}

The literature search made it possible to produce a table shows some of the devices designed by different universities and companies in the over the last twenty years, in chronological order of development. 
Table 1. Some devices designed from 2001 to today.

\begin{tabular}{|c|c|c|c|}
\hline Device name & $\begin{array}{l}\text { Year of } \\
\text { development }\end{array}$ & Developers & Type of assistance \\
\hline BLEEX & 2001 & $\begin{array}{l}\text { Berkeley Robotics and } \\
\text { Human Engineering } \\
\text { Laboratory }\end{array}$ & $\begin{array}{l}\text { Allows the movement of the limb with } \\
\text { greater force and resistance }\end{array}$ \\
\hline LOKOMAT & 2001 & Hocoma Society & $\begin{array}{l}\text { Relieves the weight body and maintains } \\
\text { standing the patient on a conveyor belt } \\
\text { while the robot reproduces the pattern } \\
\text { of the pass }\end{array}$ \\
\hline EKSO & 2005 & Ekso Bionics Society & $\begin{array}{l}\text { It improves the strength, the mobility } \\
\text { and the resistance of subject }\end{array}$ \\
\hline REWALK & 2008 & $\begin{array}{l}\text { Argo Medical } \\
\text { Technologies }\end{array}$ & $\begin{array}{l}\text { Allows you to stay in standing at people } \\
\text { with injury medullary, the gait, of go up } \\
\text { and down the stairs }\end{array}$ \\
\hline eLEGS & 2010 & Berkeley Bionics & $\begin{array}{l}\text { Allows the locomotion, to rise and down } \\
\text { the stairs, is useful in rehabilitation }\end{array}$ \\
\hline ANDROS & 2011 & $\begin{array}{l}\text { Harvard Medical } \\
\text { School e Spaulding } \\
\text { Rehabilitation Hospital }\end{array}$ & Rehabilitates gait \\
\hline HERCULE & 2011 & RB3D Society & $\begin{array}{l}\text { Exoskeleton worn by soldiers, also } \\
\text { useful for people disabled }\end{array}$ \\
\hline REX & 2012 & $\begin{array}{l}\text { Richard Little and } \\
\text { Robert Irving }\end{array}$ & $\begin{array}{l}\text { It allows you to get up, to stand, climb } \\
\text { the stairs, advance on various surfaces, } \\
\text { uphill or downhill }\end{array}$ \\
\hline HAL 3 - HAL 5 & 2012 & $\begin{array}{l}\text { Japanese University of } \\
\text { Tsukuba and } \\
\text { Cyberdyne Company }\end{array}$ & $\begin{array}{l}\text { Hal } 3 \text { is an exoskeleton for the sole legs } \\
\text { Hal } 5 \text { is an exoskeleton for legs, arms, } \\
\text { torso }\end{array}$ \\
\hline LOPES & 2012 & University of Twente & $\begin{array}{l}\text { Allows a better movement of legs and a } \\
\text { therapy less rigid }\end{array}$ \\
\hline $\begin{array}{l}\text { EXOSKELETON } \\
\text { OF VANDERBILT }\end{array}$ & 2012 & $\begin{array}{l}\text { Vanderbilt University, } \\
\text { Nashville, Stati uniti }\end{array}$ & Assistance to human locomotion \\
\hline ALEX & 2013 & University of Delaware & Exoskeleton for the motor rehabilitation \\
\hline HEXAR & 2014 & $\begin{array}{l}\text { Hanyang University of } \\
\text { Seoul }\end{array}$ & Enhance the capabilities Physical \\
\hline MINDWALKER & 2014 & $\begin{array}{l}\text { Delft University of } \\
\text { Technology }\end{array}$ & Patient care paraplegic \\
\hline INDEGO & 2014 & $\begin{array}{l}\text { Parker Hannifin } \\
\text { Corporation }\end{array}$ & $\begin{array}{l}\text { Allows the independence of people with } \\
\text { problems of mobility }\end{array}$ \\
\hline CUHK-EXO & 2015 & $\begin{array}{l}\text { Chinese University of } \\
\text { Hong Kong }\end{array}$ & Assistance to human locomotion \\
\hline PHOENIX & 2016 & MES Society & $\begin{array}{l}\text { Addressed to all the people who have } \\
\text { lost the use of legs due to traumatic } \\
\text { events, neoplasms, diseases } \\
\text { neurodegenerative }\end{array}$ \\
\hline
\end{tabular}




\section{DISCUSSION}

The exoskeleton represents one of the most alternative and innovative methods for rehabilitating people with neurodegenerative diseases, such as stroke, Parkinson's, Multiple Sclerosis and people who have suffered accidents involving the motor centres.

This device allows you to re-educate and restore walking, making it more fluid and coordinated; it does not replace the operator, but assists and intensifies his work, some models include the presence of a professional and external aids, such as crutches for example, to assist and balance walking.

It is known how hard the walk is in people with neurodegenerative diseases; the step appears unstable and insecure, not autonomous. In addition, joint mobility is reduced and muscle trophism prevails.

Improving lower extremity function and, if possible, recovering the ability to walk are important priorities for individuals who have lost locomotor function and are typically a focus of rehabilitation therapies (Read et al., 2020).

\section{Learning to walk again is one of the main goals of rehabilitation}

Walking is a rhythmic and sequential succession of flexion and extension movements, by the lower limbs, which allow the body to advance along a line of progression, maintaining balanced posture.

Pathological conditions alter the mode and efficiency of walking, with deficits affecting stability, loss of control and progression. This is caused by the control of gait, which is a cyclic movement, controlled by the cortical and subcortical areas and a lesion of these, causes an impairment in locomotion.

The motor control and the complexity of movements that undergo alteration in conditions of neurodegenerative diseases, require a "guide" that allows motor recovery by influencing the neurobiology of neuronal plasticity, providing controlled, repetitive and variable patterns; this is entrusted to rehabilitation.

Among the robotic devices used in rehabilitation, there are those with an exoskeletal system, in which there is an interaction between the robot and the human user, in which the rehabilitation therapy is guided along a pre-programmed trajectory.

The exoskeleton is an excellent aid for walking's rehabilitation, as it has different assistance settings, which can be entrusted to the therapist or to the subject himself.

\section{Robotic exoskeletons provide an external source of controlled joint power}

Compared to conventional walking training, robotic exoskeletons (such as Ekso) have been shown to dramatically increase the amount of work that can be done by the lower limbs, representing a revolution in rehabilitation. (Fleerkotte et al., 2014).

It's critical to identify the differences between exoskeletal walking and normal walking before using a robotic exoskeletal system for gait training.

From a study conducted in the United States, in particular on the EKSO exoskeleton (Swank et al., 2019), significant differences were found in the involvement of the muscle groups of the lower limbs bilaterally, as well as in gait with and without exoskeleton. 
In the stance phase, using EKSO, the movements of the hip and ankle were less, while the movements of the knee on both lower extremities were greater. In the swing phase, significant differences were found in knee and ankle movements rather than hip movements.

In particular, in the swing phases, walking with EKSO produced equivalent but lower hip movements for those of the knee and ankle, with significant differences between EKSO conditions and without, only for the distal muscle groups (bilateral soleus and anterior tibial) and for the medial hamstrings.

It should be emphasized that this is always a robotic unit with obvious technological limitations and, moreover, the subjects involved in the study were not previously "trained" to walk with EKSO.

Research has shown significant physical benefits for patients using the Ekso device, including improved balance, reduced spasticity and increased core muscle tone; walking training sessions using Ekso resulted in a significant increase in walking time and number of steps, with an increase in strength and postural control in patients with "poststroke" hemiplegia.

Rehabilitation with exoskeleton can be divided into three phases:

- First step: the operator, to allow the patient to become familiar with the tool, checks each step and the beginning of each phase, activating it manually;

- Active Step: the user controls the start of each step through an interface, placed on the crutches or on the walker, which communicates with the device;

- Pro Step: the user controls the start of the next phase by moving the hips forward and sideways; the device, thanks to sensors with advanced technology, recognizes that the patient is in the correct position and allows the step of the contralateral leg (Holanda et al., 2017).

The more advanced exoskeletons can, depending on the severity of the patient's physical condition and his response to stimuli, as well as on the brain lesions present, provide for assisted re-education.

There are different types of assistance, fixed or adaptive, bilateral or unilateral assistance, or, the walker, may not even provide any assistance. (De Luca et al., 2019).

Bilateral assistance provides full position stability and swing assistance (fixed or adaptive) to both legs. Unilateral assistance only provides them for a selected limb, while the contralateral leg is automatically released, meaning users voluntarily control the swing of that leg with their own strength and coordination, without any help from the device.

Another type of assistance is fixed assistance, in which a "fixed" intensity value is attributed during the gait oscillation phase, which can be equal to $0 \%$, if the device does not provide assistance and the subject must move actively or have a value of $100 \%$ and therefore be practically "auxiliary-dependent" for walking, without any voluntary contribution.

The benefits of using exoskeletons are undisputed, which can be used both as rehabilitation aids, as assistance tools and therefore as orthoses.

These can replace patients deficient motor function, imparting greater strength to regain the ability to perform the essential movements of daily life such as standing, sitting and walking. 


\section{A sedentary lifestyle increases the risk of chronic diseases}

For example, the metabolic syndrome (MS) is characterized by dysorlipidemia, hypertension, glucose intolerance, and obesity, which, when all factors are present, it may be a risk for developing cardiovascular or coronary disease.

Sedentary behaviour refers to activities that involve energy expenditure at the level of 1.0-1.5 metabolic equivalent units (METs). Sedentary behaviour is not simply low levels of physical activity but it can be referred to as 'sitting time'.

The effect of exercise on the body is multiple, it also increases GLUT4 content, glycogen synthase activity, mitochondrial enzyme activity improves endothelial function, may alter muscle fibre type and improves insulin sensitivity over the long term.

Regular physical activity enhances health and physical fitness improving overall the quality of life in elderly population by reducing the risk of deterioration of functional capacity. (Di Maio et al., 2020).

Moreover, the osteogenic adaptation of skeleton is site-specific and related to training regimens.

Changes of gravitational conditions can be produced also by varying magnitude and frequency of mechanical stimulus, for example, with mechanical vibrations applied to the whole body.

In fact, the first clinical studies on human being show a positive effect in adolescents with cerebral palsy and osteoporotic female. (Foti et al., 2009).

Therefore, through movement, both the style and the quality of life of these patients improve; and not only with regard to walking, but also for the symptoms of the patient himself, who manifests a reduction of pains spread above all on the lower limbs and on the rachis.

The possibility of taking advantage of assistance by exoskeleton is also of great support for the elderly, who make up a large part of the population around the world.

In different applications, carriers have different physical conditions and therefore different control strategies should be adopted.

Generally, the elderly use various aids and supports such as crutches which help them maintain balance, walk.

One major drawback of using crutches is that it requires the involvement of healthy upper limbs.

The robotic exoskeletons, with their movement, are able to provide assistance during walking by exerting additional forces that compensate for the weakness of both limbs of the elderly subject.

When crutches and walkers are not enough to support people with walking difficulties, wheelchairs are considered one of the safest tools for getting around. 
However elderly people, who usually have poor health conditions, are unable to carry out normal daily activities and, if the person spends a lot of time sitting, inactivity can lead to worsening of the general health conditions; which is why wheelchairs are no longer an effective aid for the elderly.

Furthermore, one of the most frequent aspects in these subjects is the instability and fall that affect a third of people over the age of 65 .

One of the most common consequences after the fall are bone fractures of the upper and lower limbs or both, with possible and possible injuries and trauma to the head.

An alternative to wheelchairs can now be exoskeletons, useful tools to allow and assist the elderly to move more freely and easily. Furthermore, they allow greater autonomy, with a more fluid and controlled gait, allowing you to carry out daily activities with less difficulty, thus having a positive social impact (Foti et al., 2009).

For this reason, various robotic devices have been created, aimed mainly at elderly people, previously mentioned in the METHOD and RESULTS paragraph.

Weight, space limitation and value for money should all be considered in the design of an exoskeleton to make the facility more easily accessible.

The aim is to provide intelligent assistance and to do so, it's essential to acquire different types of data related to movement. This data is used to recognize the intention of the movement of the subject wearing the exoskeleton. The subject and the exoskeleton form a closed circuit, a system of cooperation between man and machine.

Rehabilitation with robotic devices in hemiplegic patients or patients suffering from other neurodegenerative diseases, has greater efficiency and effectiveness than traditional rehabilitation, in terms of motor control and evaluation of functional results.

Researchers and experts are convinced that, over time, the exoskeleton will eventually replace the wheelchair for good.

\section{CONCLUSIONS}

With the populations of industrialized societies progressively approaching aging and with the increase in the incidence of neurodegenerative diseases and cases of stroke, there is no doubt that exoskeletons represent a solution, for rehabilitation and for the performance of daily tasks, that will make a difference in the lives of people who will have the opportunity to use it.

For this reason, however, it will be necessary to focus on certain features that increase the performance and accessibility of these devices (Bing Chen et al., 2015).

To design an effective, stable, low-mass and economical exoskeleton, research should focus on some aspects, such as: 
Weight: the exoskeletons should be as light as possible; the frame and the components add-ons shouldn't burden the user too much; materials such as the carbon fibre lend itself well to this improvement. Even the use of printers three-dimensional to achieve some elements of the exoskeleton could be suitable for purpose.

Actuators: should have a small volume, a high power / weight ratio, high efficiency and compliance, and their duration should be increased over time, maintaining initial performance. In the future, actuators will probably be developed pneumatic muscles, easily manufactured, which use a principle similar to that of natural skeletal muscles and which will certainly improve the performance of the exoskeletons, especially those used in rehabilitation applications.

Human-exoskeleton interface: the device should adapt to the patient's needs, that is, be highly customizable. Exoskeletons are worn by users via straps and padding that often cause skin irritation problems; to improve the "Wearability" of the devices, a fundamental innovation must concern the contact points between the body and the exoskeleton in order to prevent any dermatological problems. Even the size should be adjustable, to fit each individual. At present the exoskeletons are still not smart enough to help the wearer move in the desired way and direction. This factor is due to the lack of a correct exchange of information between the wearer of the exoskeleton and his nervous system.

It would be optimal that, in the future, exoskeletal control systems could use i EMG signals from sensors placed inside the muscle, so as to evaluate correctly and quickly the motor intention of the individual.

Neural implants are also an option that can be used in the future; an electroencephalogram (EEG) based interface could be developed for check the exoskeletons if limitations such as high sensitivity will be overcome e superposition of different electrical activities generated by different cortical areas.

Safety: Additional control strategies should be implemented to ensure safety of those who use the exoskeleton even in emergency conditions, such as for example physical stop systems and automatic shutdown systems. The safety of the power supply battery must be taken into consideration.

Energy: the duration of the exoskeleton power supply should be improved for prolonging the operating time; however, this is in contrast to the decrease in weight of the batteries which is essential.

Cost: robotic devices have a high cost; this makes purchasing difficult for many subjects who need it but who cannot afford it. With the improvements of robotics and technologies, it is desirable that the price of actuators and sensors to high performance can be lowered to make the exoskeleton system more accessible.

If in the near future the performance of the exoskeletons is implemented and improved, such devices, which represent a valid contribution in the field of rehabilitation and recovery motor, will become fundamental and widely used tools among the subjects who will have the need for movement supports.

\section{REFERENCES}

Chen, B., Ma, H., Qin, L. H., Gao, F., Chan, K. M., Law, S. W., Qin, L., \& Liao, W. H. (2015). Recent developments and challenges of lower extremity exoskeletons. Journal of Orthopedic Translation. Hong Kong, China. https://doi.org/10.1016/i.jot.2015.09.007

De Luca, A., Bellitto, A., Massone, A., Leoncini, C., Marchesi, G., Pellegrino, L., Rossi, L., Coscia, M., Casadio, M., Mandraccia, S., \& Gamba, S. (2019). Exoskeleton for Gait Rehabilitation: Effects of 
Assistance, Mechanical Structure, and Walking Aids on Muscle Activations. Genoa, Italy. https://doi.org/10.3390/app9142868

Di Maio, G., Monda, V., Messina, A., Polito, R., Monda M., Tartaglia, N., Ambrosio, A., Pisanelli, D., Asmundo, A., Di Nunno, N., Ametta, A., Villano, I., \& Francavilla, V. C. (2020). Physical Activity and modification of lifestyle induce benefits on the health status. Acta Medica Mediterranea. http://dx.doi.org/10.19193\%2F0393-6384_2020_3_299

Fleerkotte, B. M., Koopman, B., Buurke, J. H., van Asseldonk, E. H., van der Kooij, H., \& Rietman, J. S. (2014). The effect of impedance-controlled robotic gait training on walking ability and quality in individuals with chronic incomplete spinal cord injury: an explorative study. Journal of NeuroEngineering and Rehabilitation. https://doi.org/10.1186/1743-0003-11-26

Foti, C., Annino, G., D'Ottavio, S., Sensi, F., Tsarpela, O., Masala, S., Magni, E., Tranquilli, C., Francavilla, C., \& Bosco, C. (2009). Preliminary study on the effects of high magnitude, low frequency of whole body vibration in physical activity of osteoporotic woman. Orthopedic Area.

Holanda, L. J., Silva, P. M., Amorim, T. C., Lacerda, M. O., Simão, C. R., \& Morya, E. (2017). Robotic assisted gait as a tool for rehabilitation of individuals with spinal cord injury: a systematic review. Journal of Neuroengineering and Rehabilitation, vol. 14, no. 1, p.126. https://doi.org/10.1186/s12984-017-0338-7

Kapsalyamov, A., Jamwal, K.P. (2019). State of the Art Lower Limb Robotic Exoskeletons for Elderly Assistance", Journal of Orthopaedic Translation. https://doi.org/10.1109/ACCESS.2019.2928010

Molteni F., Gasperini, G., \& Cannaviello, G. (2018). Exoskeleton and end-effector robots for upper and lower limbs rehabilitation: narrative review. Journal of NeuroEngineering and Rehabilitation, vol. 11, no. 1, p. 26. https://doi.org/10.1016/j.pmri.2018.06.005

Read, E., Woolsey, C., McGibbon, C. A., \& O'Connell, C. (2020). Physiotherapists' Experiences Using the Ekso Bionic, Exoskeleton with Patients in a Neurological Rehabilitation Hospital: A Qualitative Study, Rehabilitation Research and Practice. Rehabilitation Research and Practice. https://doi.org/10.1155/2020/2939573

Simeonov, S., Veneva, I., (2019) Active Orthoses of the lower limbs. Survey and Preliminary Investigation, Rehabilitation Robotics.

Swank, C., Wang-Price, S., Gao, F., \& Almutairi, S. (2019). Walking With a Robotic Exoskeleton Does Not Mimic Natural Gait: A Within-Subjects Study. JMIR Rehabilitation and Assistive Technologies, v. 6, issue 1, e11023, p. 1-10. Dallas, TX. https://doi.org/10.2196/11023

\section{(ब) $\odot \Theta$}

This work is licensed under a Attribution-NonCommercial-NoDerivatives 4.0 International (CC BY-NC-ND 4.0). 